\title{
TrueBeam Low Dose Rate Investigation for Pulsed Reduced Dose Rate IMRT
}

\author{
Mark Geurts \\ Department of Human Oncology, University of Wisconsin-Madison, Madison, WI, USA \\ Email: mwgeurts@wisc.edu
}

How to cite this paper: Geurts, M. (2017) TrueBeam Low Dose Rate Investigation for Pulsed Reduced Dose Rate IMRT. International Journal of Medical Physics, Clinical Engineering and Radiation Oncology, 6, 139-149.

https://doi.org/10.4236/ijmpcero.2017.62013

Received: April 4, 2017

Accepted: May 19, 2017

Published: May 22, 2017

Copyright (C) 2017 by author and Scientific Research Publishing Inc. This work is licensed under the Creative Commons Attribution-NonCommercial International License (CC BY-NC 4.0). http://creativecommons.org/licenses/by-nc/4.0/

\begin{abstract}
The capability of the TrueBeam treatment system to deliver step and shoot IMRT plans at low dose rates was evaluated. Beam characteristics during low dose rates (5 to $100 \mathrm{MU} / \mathrm{min}$ ) were evaluated for consistency using a planar ion chamber array. MU constancy, linearity, and beam profile symmetry were all found to be equivalent within $0.5 \%$. The response of the Scandi Dos Delta 4 system was also evaluated at low dose rates of using static open beams compared to ion chamber measurements, and step and shoot IMRT plans comparing $5-20 \mathrm{MU} / \mathrm{min}$ and $100 \mathrm{MU} / \mathrm{min}$ dose rates, with a maximum observed absolute dose difference of $0.8 \%$ and equivalence margin of $0.2 \%$. The Gamma Index and measurement reproducibility were also found to be equivalent.
\end{abstract}

\section{Keywords}

PRDR, Low Dose Rate, Delta 4, TrueBeam

\section{Introduction}

Pulsed reduced dose-rate radiotherapy (PRDR) has become a powerful treatment for recurrent diseases, which comprise approximately $15 \%-20 \%$ of treatments [1] [2] [3]. Introduced as an extension of continuous low dose rate therapies, PRDR combines the advantages of accelerator-based delivery and low dose hyper-radio-sensitivity by separating conventional dose fractions into $0.2 \mathrm{~Gy}$ pulses separated by three-minute intervals to achieve a time averaged dose rate of $0.0667 \mathrm{~Gy} / \mathrm{min}$ [4] [5]. This hyper-radio-sensitivity is caused by accumulation of tumor cells in the G2 and M phases of the cell cycle during treatment.

Due to the complex nature of these treatments, PRDR using 3D-conformal techniques can be very difficult. Often patients have been previously irradiated; as such, nearby critical structures may have already received at or close to their 
toxicity limit. Researchers are actively investigating ways to apply PRDR to more advanced techniques, such as static field Intensity Modulated Radiation Therapy (IMRT) [6], Volumetric Modulated Arc Therapy (VMAT) [7] [8], and tomotherapy [9] [10]. The primary challenge with IMRT-based PRDR, however, is that IMRT fields are difficult to separate into 0.2 Gy pulses. Currently available treatment planning systems do not include optimization constraints that specifically limit dose rate. Instead, treatments must be split into multiple (often ten or more) beams or arcs each delivering less than $0.2 \mathrm{~Gy}$, or the same beams/arcs must be delivered repeated times with each sub-fraction less than $0.2 \mathrm{~Gy}$ [7].

When delivering PRDR treatments on the Varian ${ }^{\circledR}$ TrueBeam $^{\text {tw }}$ system (Varian Medical Systems, Palo Alto, CA), an alternative technique can be used to reduce the time averaged dose rate. Instead of splitting the total dose into $0.2 \mathrm{~Gy}$ pulses, a lower repetition rate can be used to achieve a time averaged dose rate of $0.0667 \mathrm{~Gy} / \mathrm{min}$. The TrueBeam system can run as low as $5 \mathrm{MU} / \mathrm{min}$. This greatly simplifies treatment planning of PRDR cases, as conventional (normal fractionation) beam arrangements and optimization techniques can be used. After optimization, the dose from each beam to the target can be computed and the dose rate adjusted during treatment delivery to achieve the desired target dose rate.

For VMAT and sliding window IMRT techniques, reducing the repetition rate will significantly slow down the gantry and MLC leaf motion, respectively. Even with static field step and shoot-type plans, the low repetition rate may affect the operation of the servo mechanisms that maintain beam stability parameters such as flatness, symmetry, and dose per MU. Typical IMRT commissioning practices also may not specifically include testing delivery at these low repetition rates [11].

Furthermore, performing patient specific plan delivery Quality Assurance (QA) at such a low repetition rate can also be problematic. Jursinic et al. [12] demonstrated that some diodes display a reduced sensitivity relative to ion chamber measurements as the dose rate is reduced. Ahmed et al. [13] conducted a similar experiment on a TrueBeam system and Sun Nuclear ${ }^{\circledR}$ ArcCHECK $^{\circledR}$ measurement phantom (Sun Nuclear Corporation, Melbourne FL), finding that the ArcCHECK under-responded by $-2.2 \%$ relative to ion chamber measurements at $5 \mathrm{MU} / \mathrm{min}$. This adds additional challenges when commissioning a PRDR program; prior to performing end-to-end testing on an IMRT QA phantom, users must first re-validate their QA system at these repetition rates and factor their devices' limitations into their results.

After commissioning is completed, there is an additional logistical concern as well. Conducting PRDR IMRT delivery QA measurements at the true low dose rate requires at least 30 minutes per run. Plans often require more than one measurement as well (either for troubleshooting or to increase the detector spatial frequency/area), making it difficult to conduct these measurements after hours in a busy clinic.

The purpose of this study was to demonstrate a procedure by which other clinicians could follow to develop PRDR at their clinics. The procedure starts with 
a series of tests to validate the delivery system at low repetition rates, then to characterize the performance of the IMRT QA system, and finally a series of end-to-end tests of patient plans that establish equivalence between IMRT delivery at PRDR and conventional repetition rates. To the author's knowledge, this is also the first reported characterization of the ScandiDos Delta $4^{\circledR}$ phantom (ScandiDos AB, Uppsala Sweden) at low repetition rates.

\section{Methods}

All measurements were performed on the TrueBeam 2.5 treatment system using the conventional flattened $6 \mathrm{MV}$ beam. IMRT QA phantom measurements were acquired on the Delta4 phantom and October 2016 software. Where stated that measurements were performed at each dose rate, acquisitions were repeated at 5 , $10,15,20,40,60,80,100$, and $600 \mathrm{MU} / \mathrm{min}$. Unless otherwise noted, static beams were delivered using a $20 \times 20 \mathrm{~cm}^{2}$ open field, IEC $0^{\circ}$ gantry/collimator positions, with the device set up to $100 \mathrm{~cm}$ Source to Surface Distance (SSD) using the optical distance indicator and aligned to the treatment system central axis using the light field, verified by the lasers.

\subsection{Beam Characteristic Equivalence}

Monitor unit dose, linearity, and beam flatness/symmetry reproducibility with dose rate were evaluated using the Sun Nuclear IC Profiler ${ }^{\mathrm{rm}}$ device and v3.4.2 software. The IC Profiler was chosen as it has been previously demonstrated to be equivalent to water tank measurements detecting changes in these metrics [14]. The treatment system was then configured to deliver $50 \mathrm{MU}$ at each dose rate. For each measurement, the central axis integral counts, IEC X/Y area symmetry, and diagonal flatness, statistics were computed and recorded.

The area symmetry SYMA is defined by Equation (1) [15], where $S A R$ and $S A L$ are the trapezoidal integrated area under the left and right central $80 \%$ field, respectively:

$$
S Y M A=\left(\frac{S A R-S A L}{S A R+S A L}\right) \times 200
$$

The diagonal flatness DFLAT is defined as the average dose at $80 \%$ of the field width along each diagonal sub-axis normalized by the central axis [15], defined by Equation (2) where $C C_{i}^{d}$ is the response at distance $d$ from the central axis along diagonal sub-axis $\dot{i}$ :

$$
D F L A T=\frac{\sum_{i=1}^{4} C C_{i}^{d}}{4 C C^{0}} \times 100
$$

The symmetry and flatness statistics from 5 to $100 \mathrm{MU} / \mathrm{min}$ tested were tested for equivalence to their respective $600 \mathrm{MU} / \mathrm{min}$ values using a paired Two One-Sided Test (TOST) by Schuirmann [16] and Westlake [17]. The robust t-test of Yuen and Dixon [18] [19] was used as it makes no assumption of normality. An equivalence margin of $0.5 \%$ was used. In addition, the results were tested for linear dependence with dose rate using an Iterative re-Weighted Least 
Squares (IWLS) robust regression was performed using an MM-type estimator and Tukey bi-square [20] re-descending score function described in Yohai [21] and Koller and Stahel [22].

Next, an IBA FC65-P Farmer chamber (Ion Beam Applications SA, Louvain-la-Neuve Belgium) was placed at $1.5 \mathrm{~cm}$ depth in solid water. The chamber was then connected to a Standard Imaging ${ }^{\circledR}$ SuperMax electrometer (Standard Imaging Inc., Middleton, WI). The collected charge was recorded for 5, 10, 15, 25 , and $50 \mathrm{MU}$ delivery using a $20 \times 20 \mathrm{~cm}^{2}$ open field, $0^{\circ}$ gantry, and $5 \mathrm{MU} / \mathrm{min}$ dose rate and corrected for temperature and pressure. The MU linearity residual errors were computed as the observed relative corrected charge minus the predicted value given a linear fit through $50 \mathrm{MU}$. The standard error was computed as the root mean square of all residuals.

\subsection{Delta 4 Static Beam Dose Rate Equivalence}

Delta 4 response reproducibility with dose rate was evaluated by comparing the Delta 4 response relative to an ionization chamber. Using the same ionization chamber setup as before, the collected charge was recorded for $50 \mathrm{MU}$ at each dose rate. The measurements were then repeated with the Delta 4 aligned to isocenter. For each measurement, mean dose across the central $6 \times 6 \mathrm{~cm}^{2}$ detectors was recorded and divided by the ionization chamber response. The ratios were then normalized to $600 \mathrm{MU} / \mathrm{min}$ and tested for equivalence and linear dependence as before using a $0.2 \%$ equivalence margin. Verification of MU linearity was also repeated with the Delta 4.

\subsection{Delta 4 Step and Shoot IMRT Equivalence}

To validate the equivalence of Delta 4 IMRT QA results with dose rate, three PRDR static step and shoot IMRT QA plans were delivered at low dose rates and $100 \mathrm{MU} / \mathrm{min}$. Each plan was optimized in the Pinnacle ${ }^{3}$ v9.8 Treatment Planning System (Philips Healthcare, Madison, WI). A summary of the beams in each plan are provided in Table 1. All patients were brain treatments, as this is the most common indication for PRDR at the University of Wisconsin-Madison. Plans were chosen with varying target sizes, creating a range of field sizes from 27.5 to $196 \mathrm{~cm}^{3}$. The average number of MU per segment ranged from 3.1 to 12.3 , with a mean of 4.7 MU. Dose rate ranged from 5 to $20 \mathrm{MU} / \mathrm{min}$.

Next, each plan was copied to the Delta 4 and re-calculated using a $2 \times 2 \times 2$ $\mathrm{mm}^{3}$ resolution. Each plan was then delivered to the Delta 4 at the low dose rate and at $100 \mathrm{MU} / \mathrm{min}$ and analyzed in the Delta 4 software. The right parietal lobe plan was measured both $5 \mathrm{MU} / \mathrm{min}$ and $10 \mathrm{MU} / \mathrm{min}$ to provide additional statistical power. The median absolute dose in the target region defined by diodes above $50 \%$ of the maximum dose and mean Gamma index [23] using 2\% (of maximum dose), $2 \mathrm{~mm}$ distance to agreement, and $20 \%$ threshold criteria were then recorded. The dose difference and mean Gamma at low and high dose rates were tested for equivalence using a paired TOST, with equivalence margins of $0.2 \%$ and 0.05 , respectively. 
Table 1. Summary of step and shoot IMRT plan parameters.

\begin{tabular}{|c|c|c|c|c|c|c|}
\hline Area Treated & Beam & Gantry & Field Size & $\mathrm{CPs}$ & $\mathrm{MU}$ & Dose Rate \\
\hline \multirow{8}{*}{ Anterior brain } & 01 Vertex & $140^{\circ}$ & $14.5 \times 13.0 \mathrm{~cm}^{2}$ & 13 & 74 & $20 \mathrm{MU} / \mathrm{min}$ \\
\hline & $02 \mathrm{LAO}$ & $55^{\circ}$ & $14.0 \times 14.5 \mathrm{~cm}^{2}$ & 10 & 74 & $10 \mathrm{MU} / \mathrm{min}$ \\
\hline & 03 LPO1 & $100^{\circ}$ & $14.5 \times 14.0 \mathrm{~cm}^{2}$ & 11 & 117 & $10 \mathrm{MU} / \mathrm{min}$ \\
\hline & 04 LPO2 & $135^{\circ}$ & $10.0 \times 14.5 \mathrm{~cm}^{2}$ & 11 & 39 & $15 \mathrm{MU} / \mathrm{min}$ \\
\hline & $05 \mathrm{PA}$ & $180^{\circ}$ & $13.0 \times 14.0 \mathrm{~cm}^{2}$ & 12 & 41 & $20 \mathrm{MU} / \mathrm{min}$ \\
\hline & 06 RPO1 & $220^{\circ}$ & $13.5 \times 14.0 \mathrm{~cm}^{2}$ & 11 & 41 & $15 \mathrm{MU} / \mathrm{min}$ \\
\hline & 07 RPO2 & $260^{\circ}$ & $12.9 \times 14.0 \mathrm{~cm}^{2}$ & 15 & 104 & $15 \mathrm{MU} / \mathrm{min}$ \\
\hline & $08 \mathrm{RAO}$ & $300^{\circ}$ & $14.5 \times 13.5 \mathrm{~cm}^{2}$ & 6 & 74 & $20 \mathrm{MU} / \mathrm{min}$ \\
\hline \multirow{5}{*}{ Vertex brain } & $01 \mathrm{RPO}$ & $225^{\circ}$ & $7.5 \times 5.0 \mathrm{~cm}^{2}$ & 8 & 59 & $10 \mathrm{MU} / \mathrm{min}$ \\
\hline & $02 \mathrm{RAO}$ & $290^{\circ}$ & $7.0 \times 5.0 \mathrm{~cm}^{2}$ & 8 & 66 & $10 \mathrm{MU} / \mathrm{min}$ \\
\hline & $03 \mathrm{LAO}$ & $70^{\circ}$ & $7.5 \times 5.0 \mathrm{~cm}^{2}$ & 10 & 62 & $5 \mathrm{MU} / \mathrm{min}$ \\
\hline & $04 \mathrm{LPO}$ & $140^{\circ}$ & $7.5 \times 5.0 \mathrm{~cm}^{2}$ & 8 & 56 & $5 \mathrm{MU} / \mathrm{min}$ \\
\hline & 05 Vertex & $41^{\circ}$ & $5.5 \times 5.0 \mathrm{~cm}^{2}$ & 7 & 57 & $5 \mathrm{MU} / \mathrm{min}$ \\
\hline \multirow{5}{*}{ Right parietal lobe } & $01 \mathrm{PA}$ & $180^{\circ}$ & $11.0 \times 13.5 \mathrm{~cm}^{2}$ & 9 & 39 & $5,10 \mathrm{MU} / \mathrm{min}$ \\
\hline & $02 \mathrm{RPO}$ & $220^{\circ}$ & $13.0 \times 14.0 \mathrm{~cm}^{2}$ & 9 & 26 & $5,10 \mathrm{MU} / \mathrm{min}$ \\
\hline & 03 RLat & $270^{\circ}$ & $13.0 \times 15.0 \mathrm{~cm}^{2}$ & 7 & 24 & $5,10 \mathrm{MU} / \mathrm{min}$ \\
\hline & $04 \mathrm{RAO}$ & $310^{\circ}$ & $13.0 \times 13.5 \mathrm{~cm}^{2}$ & 8 & 30 & $5,10 \mathrm{MU} / \mathrm{min}$ \\
\hline & 05 Vertex & $25^{\circ}$ & $11.0 \times 12.5 \mathrm{~cm}^{2}$ & 8 & 25 & $5,10 \mathrm{MU} / \mathrm{min}$ \\
\hline
\end{tabular}

Finally, to evaluate if dose rate affects the variance in IMRT QA results the right parietal lobe field $5 \mathrm{MU} / \mathrm{min}, 10 \mathrm{MU} / \mathrm{min}$, and $100 \mathrm{MU} / \mathrm{min}$ beam measurements were each repeated three times. The standard deviation of each measurement set was then computed and tested for equivalence using the same paired TOST.

\section{Results and Discussion}

The following sections detail the results of the tests described above. Statistical analysis was conducted in R v.3.3.2 (R Foundation, https://www.r-project.org). A significance level of 0.05 (95\% confidence) was established a priori for hypothesis testing.

\subsection{Beam Characteristic Equivalence}

Figure 1 illustrates the central axis dose, diagonal flatness, and area symmetry (along $\mathrm{X}$ and $\mathrm{Y}$ axes) with dose rate. At $600 \mathrm{MU} / \mathrm{min}$, these values are consistent with peers' results from other TrueBeam clinical systems [24]. The central axis dose was found to be equivalent with doserate, $\varepsilon=0.5 \%, 95 \%$ CI $[-0.09 \%$ $0.11 \%], p<0.001$, with no linear dependence observed, $p=0.111$. The diagonal flatness was not equivalent, $\varepsilon=0.5 \%, 95 \%$ CI $[-0.91 \%-0.35 \%], p=0.795$, and instead did display a linear dependence, $p=0.023$, adj. $\mathrm{R}^{2}=0.73$. This is likely due to the increased noise in the measured profiles as the dose rate decreased; 
the $\mathrm{Y}$ axis profile at $5 \mathrm{MU} / \mathrm{min}$ is plotted (in magenta) over the other dose rate profiles in Figure 2.

Area symmetry along the $\mathrm{X}$ axis was equivalent, $\mathcal{\varepsilon}=0.5 \%, 95 \% \mathrm{CI}[-0.37 \%$ $0.08 \%], p=0.009$, although with an inverse linear relationship with dose rate, $p$ $=0.020$, adj. $\mathrm{R}^{2}=0.58$. Area symmetry along the $\mathrm{Y}$ axis was equivalent, $\varepsilon=0.5 \%$, $95 \%$ CI $[0.22 \% 0.45 \%], p=0.014$, with no statistical linear dependence, $p=$ 0.972 .

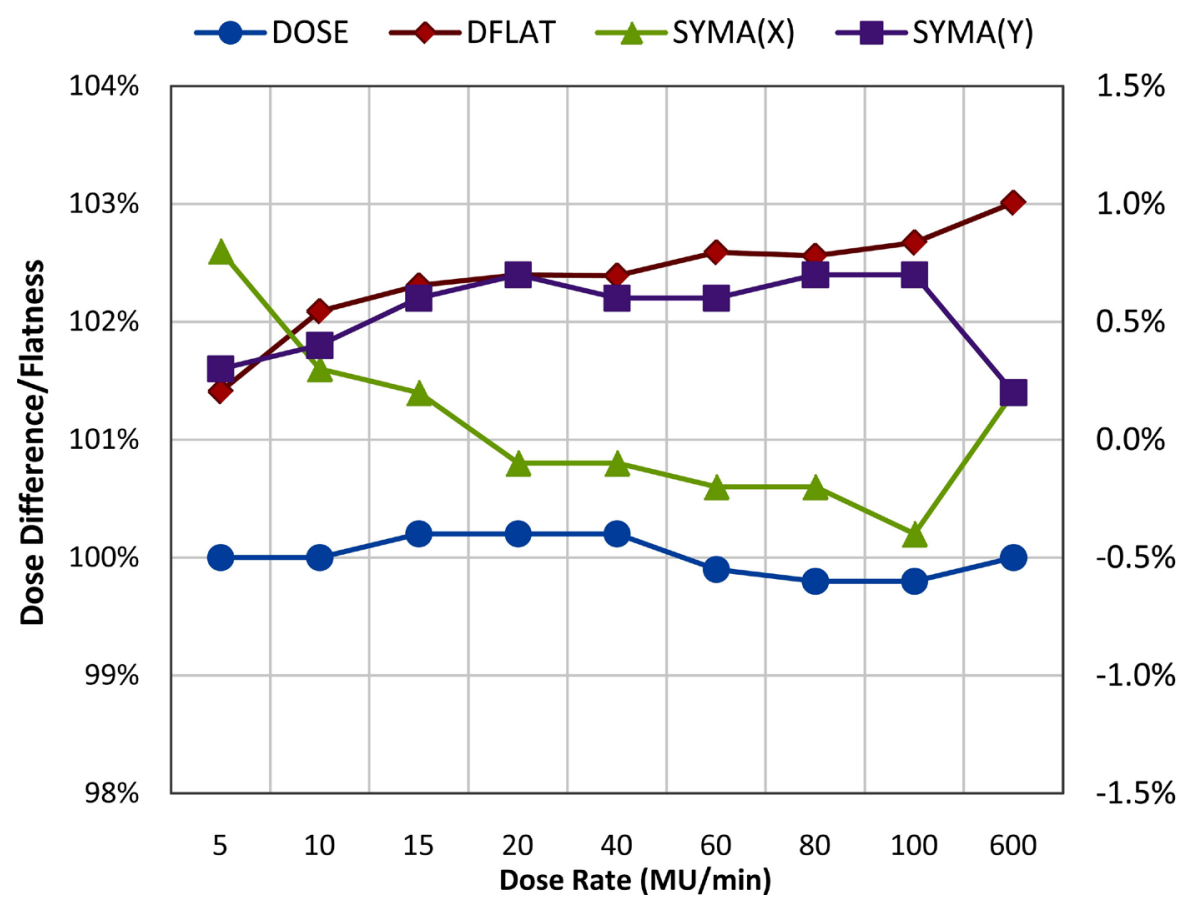

Figure 1. Beam characteristics (dose, flatness, and symmetry) with dose rate.

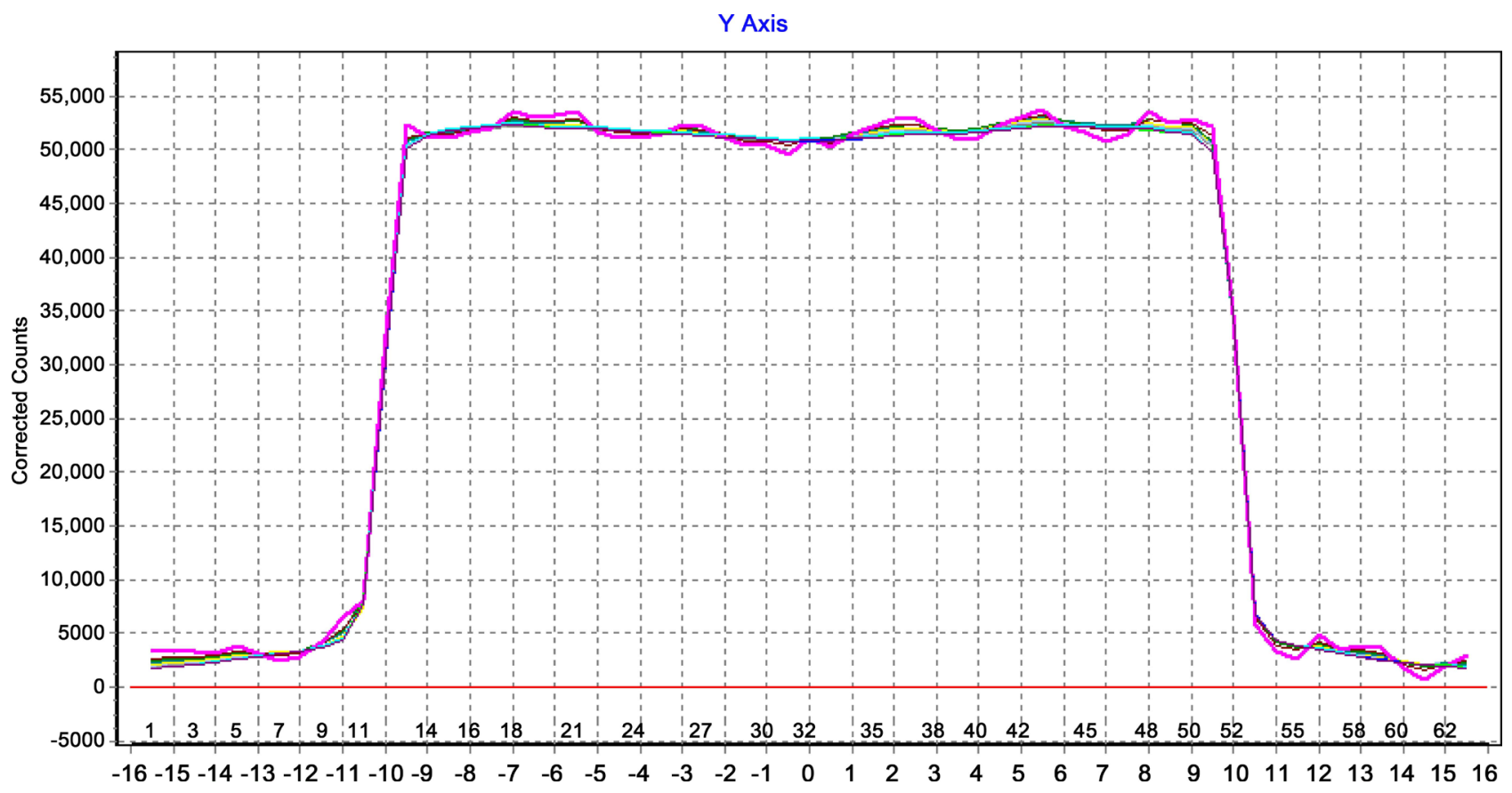

Figure 2. Beam Y axis profile at different dose rates. The magenta (noisy) profile is $5 \mathrm{MU} / \mathrm{min}$. 
Changes in symmetry with dose rate suggest that the dose servos have a slight effect on beam steering. That said, AAPM Task Group 142 [25] recommends period QA flatness and symmetry changes from baseline to not exceed $1 \%$ from baseline; at $1 \%$ equivalence margin all beam characteristics were statistically significant including flatness, $p<0.019$. Finally, for MU linearity, the residual standard and maximum errors were $0.25 \%$ and $0.55 \%$ at $5 \mathrm{MU} / \mathrm{min}$ between 5 $50 \mathrm{MU}$, respectively. The residual errors are plotted in Figure 3.

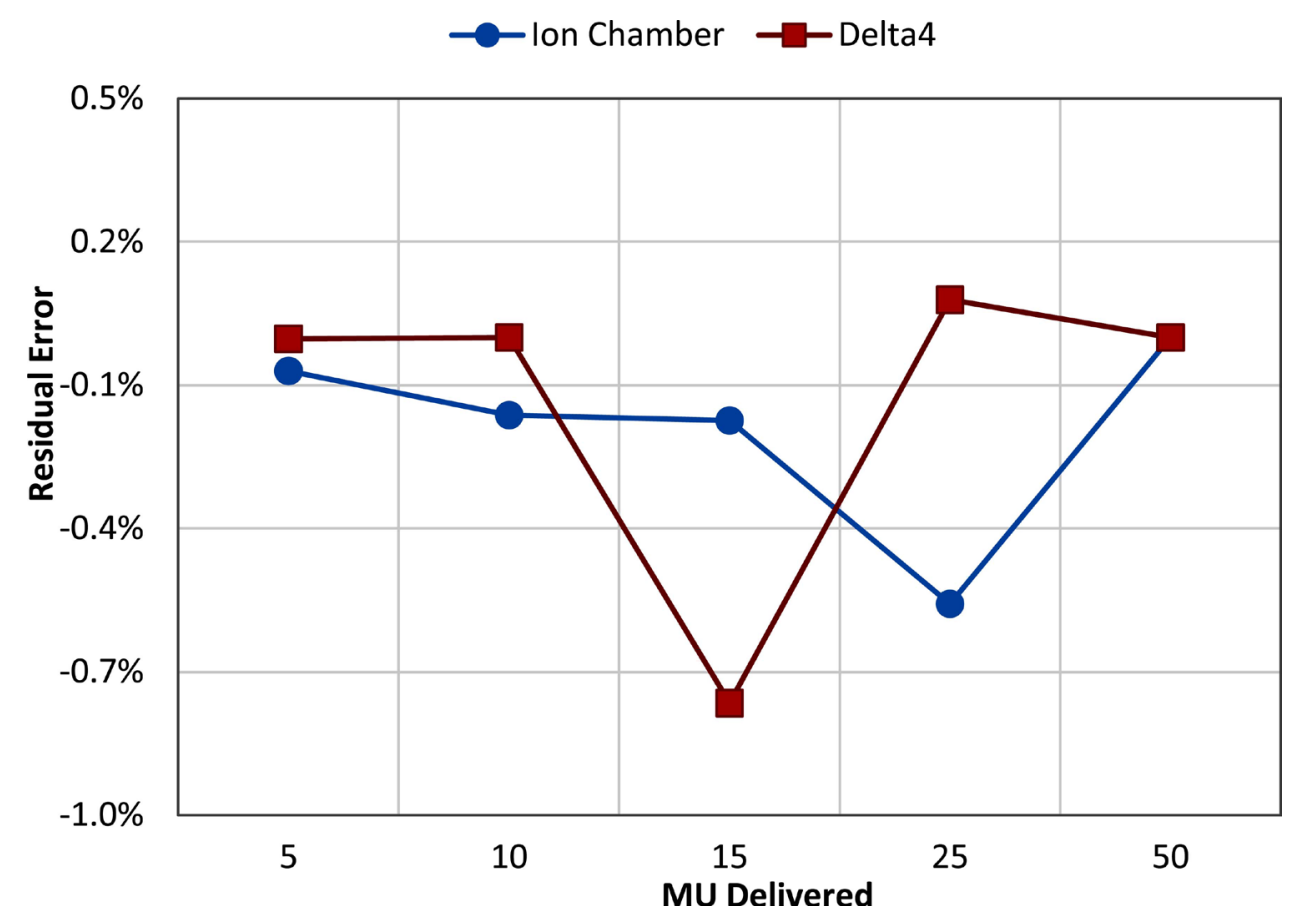

Figure 3. MU linearity residual errors for ion chamber and Delta 4.

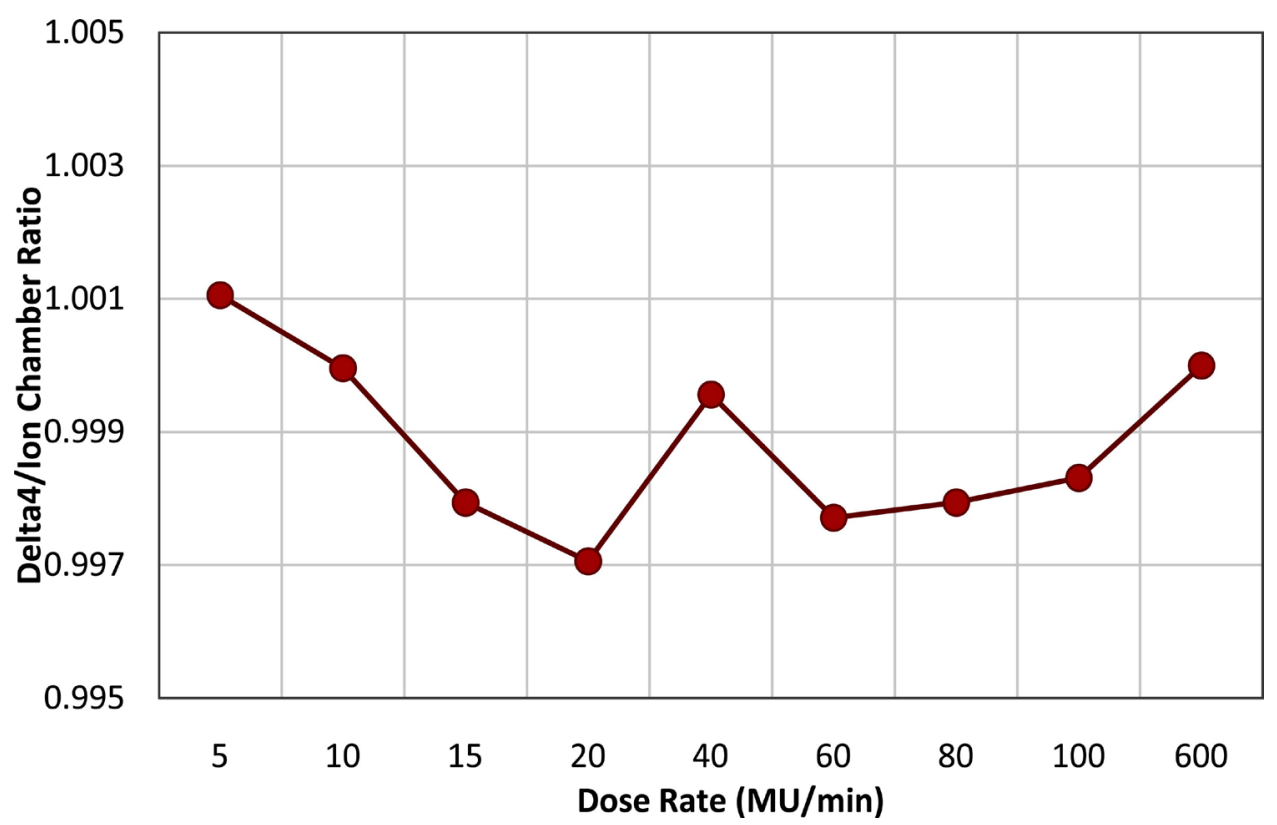

Figure 4. Delta 4 response relative to ion chamber with dose rate for a $20 \times 20$ field, normalized to $600 \mathrm{MU} / \mathrm{min}$. 


\subsection{Delta 4 Static Beam Dose Equivalence}

Figure 4 illustrates the Delta4 mean response (within the central $6 \times 6 \mathrm{~cm}^{2}$ detectors) relative to ion chamber response under the same conditions as a function of dose rate, normalized to 1.000 at $600 \mathrm{MU} / \mathrm{min}$. The low dose rates $(5-100$ $\mathrm{MU} / \mathrm{min}$ ) were found to be equivalent to $600 \mathrm{MU} / \mathrm{min}, \varepsilon=0.2 \%, 95 \% \mathrm{CI}[-0.20 \%$ $-0.03 \%$ ], $p=0.049$, as well as no linear dependence with dose rate, $p=0.763$.

These findings suggest that the Delta4 diode design does not suffer from the low dose rate under-response reported with the ArcCHECK [13] and other diodes [12]. According to the vendor, the proprietary design of the diodes was optimized to maximize response constancy with delivery parameter variability, including PRF rate (personal communication, January 2017). For MU linearity, the residual standard and maximum errors were $0.40 \%$ and $0.76 \%$, respectively. Residual errors are plotted in Figure 3.

\subsection{Delta4 Step and Shoot IMRT Equivalence}

Paired measurements (at 5 - $20 \mathrm{MU} / \mathrm{min}$ and $100 \mathrm{MU} / \mathrm{min}$ ) of each beam in Table 1 were collected for equivalence testing $(n=41)$, and are plotted in Figure 5 . The median absolute dose was found to be equivalent between the two dose rate groups, $\mathcal{E}=0.2 \%, 95 \%$ CI $[-0.015 \% 0.13 \%], p=0.001$, while the mean $2 \% / 2 \mathrm{~mm}$ Gamma index was also found to be equivalent, $\varepsilon=0.05,95 \%$ CI [-0.0034 0.029], $p<0.001$. The maximum difference in median absolute dose between groups was $0.8 \%$. IWLS robust regression yielded a significant correlation, $p<0.001$, adj. $\mathrm{R}^{2}=0.99$.

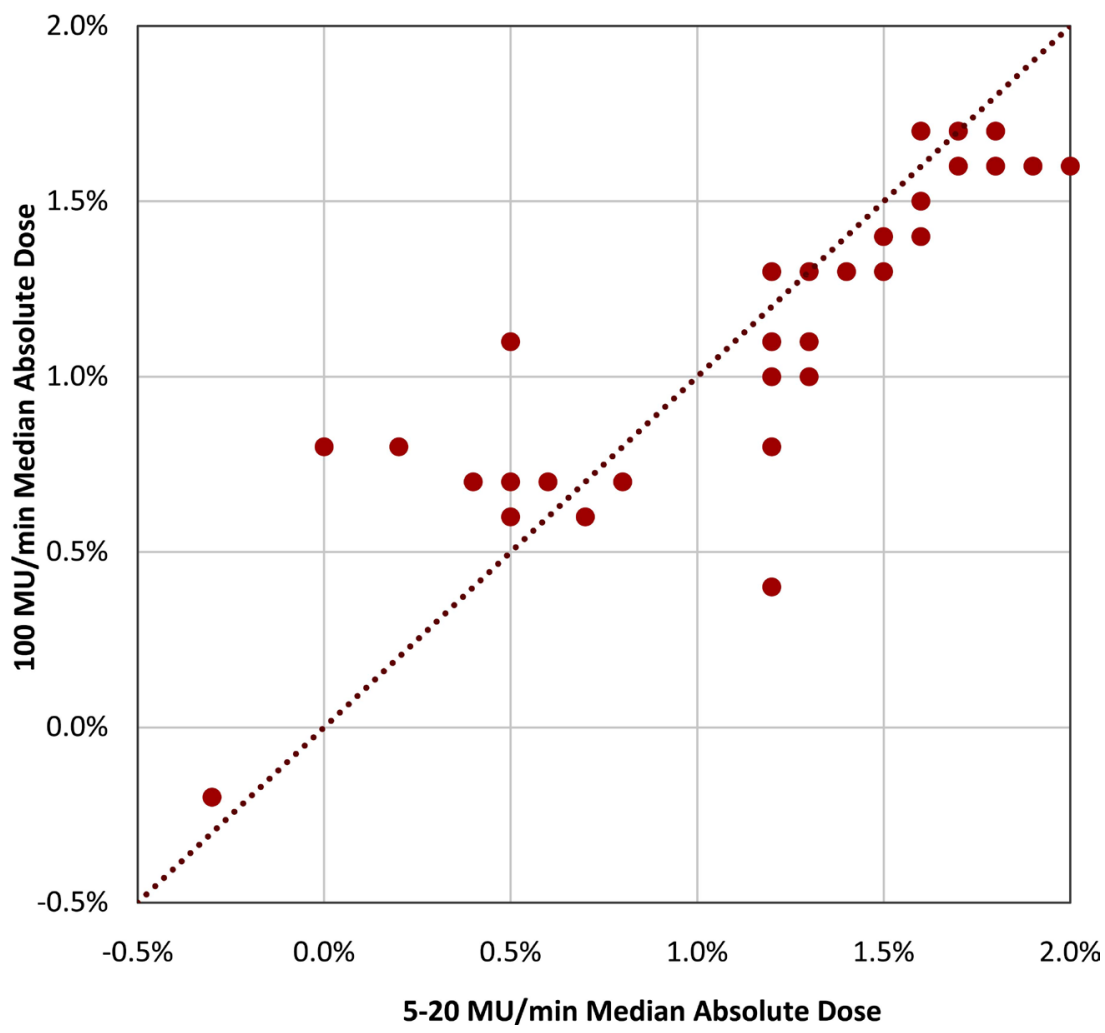

Figure 5. Scatter plot of per-field Delta 4 median absolute dose difference (relative to TPS) at 5 - $20 \mathrm{MU} / \mathrm{min}$ versus $100 \mathrm{MU} / \mathrm{min}$. The dashed line indicates identical response. 
Furthermore, the variance in measurement was also found to be equivalent between dose rate groups $(n=5)$ for the median absolute dose, $\varepsilon=0.2 \%, 95 \% \mathrm{CI}$ [-0.0056\% 0.080\%], $p<0.001$, and Gamma index metrics, $\varepsilon=0.05,95 \% \mathrm{CI}$ [-0.0084 0.025], $p=0.003$.

Finally, using the planned low dose rate, the composite median dose difference (between measured and calculated by the treatment planning system) in the target region and 3\% (of maximum dose), $3 \mathrm{~mm}, 20 \%$ threshold Gamma index met the clinic's IMRT QA acceptance criteria for all plans. The mean, minimum, and maximum median dose differences were $1.4 \%, 0.9 \%$, and $2.2 \%$, while the Gamma index pass rates were $99.3 \%, 98.2 \%$, and $100 \%$, respectively. It is notable that all PRDR plans yielded a median difference greater than one; unfortunately, not enough plans were measured to determine if the distribution of PRDR plan results statistically differ from other types of treatments. As this technique is employed at this center and more plans are created, this is an opportunity for future study.

\section{Conclusion}

This report demonstrates the capability of the TrueBeam system to deliver step and shoot PRDR IMRT plans and establishes equivalence between IMRT QA at $100 \mathrm{MU} / \mathrm{min}$ and lower dose rates. Static beam measurements found the TrueBeam system to achieve MU constancy, linearity, and symmetry within $0.5 \%$ margin. The Delta 4 phantom also responded equivalently within a $0.2 \%$ margin between ion chamber measurements for static beams and between $100 \mathrm{MU} / \mathrm{min}$ and lower dose rates for step and shoot IMRT.

\section{References}

[1] Cannon, G.M., Tomé, W.A., Robins, H.I. and Howard, S.P. (2007) Pulsed Reduced Dose-Rate Radiotherapy: Case Report: A Novel Re-Treatment Strategy in the Management of Recurrent Glioblastoma Multiforme. Journal of Neuro-Oncology, 83, 307-311. https://doi.org/10.1007/s11060-007-9329-Z

[2] Richards, G.M., Tomé, W.A., Robins, H.I., Stewart, J.A., Welsh, J.S., Mahler, P.A., et al. (2009) Pulsed Reduced Dose-Rate Radiotherapy: A Novel Locoregional Retreatment Strategy for Breast Cancer Recurrence in the Previously Irradiated Chest Wall, Axilla, or Supraclavicular Region. Breast Cancer Research and Treatment, 114, $307-$ 313. https://doi.org/10.1007/s10549-008-9995-3

[3] Adkison, J.B., Tomé, W., Seo, S., Richards, G.M., Robins, H.I., Rassmussen, K., et al. (2011) Reirradiation of Large-Volume Recurrent Glioma with Pulsed Reduced Dose Rate Radiotherapy. International Journal of Radiation Oncology, Biology, Physicss, 79, 835-841. https://doi.org/10.1016/j.ijrobp.2009.11.058

[4] Fowler, J.F., Welsh, J.S. and Howard, S.P. (2004) Loss of Biological Effect in Prolonged Fraction Delivery. International Journal of Radiation Oncology, Biology, Physics, 59, 242-249. https://doi.org/10.1016/j.ijrobp.2004.01.004

[5] Tomé, W.A. and Howard, S.P. (2007) On the Possible Increase in Local Tumour Control Probability for Gliomas Exhibiting Low Dose Hyper-Radiosensitivity Using a Pulsed Schedule. The British Journal of Radiology, 80, 32-37.

https://doi.org/10.1259/bjr/15764945 
[6] Kang, S., Lang, J., Wang, P., Li, J., Lin, M., Chen, X., et al. (2014) Optimization Strategies for Pulsed Low-Dose-Rate IMRT of Recurrent Lung and Head and Neck Cancers. Journal of Applied Clinical Medical Physics, 15, 4661. https://doi.org/10.1120/jacmp.v15i3.4661

[7] Ma, C.M., Lin, M.H., Dai, X.F., Koren, S., Klayton, T., Wang, L., et al. (2012) Investigation of Pulsed Low Dose Rate Radiotherapy Using Dynamic Arc Delivery Techniques. Physics in Medicine and Biology, 57, 4613-4626.

https://doi.org/10.1088/0031-9155/57/14/4613

[8] Tyagi, N., Yang, K., Sandhu, R., Yan, D., Park, S.S., Chen, P.Y. and Marples, B. (2013) External Beam Pulsed Low Dose Radiotherapy Using Volumetric Modulated Arc Therapy: Planning and Delivery. Medical Physics, 40, Article Number: 011704. https://doi.org/10.1118/1.4769119

[9] Rong, Y., Paliwal, B., Howard, S.P. and Welsh, J. (2011) Treatment Planning for Pulsed Reduced Dose-Rate Radiotherapy in Helical Tomotherapy. International Journal of Radiation Oncology, Biology, Physics, 79, 934-942. https://doi.org/10.1016/j.ijrobp.2010.05.055

[10] Geurts, M. (2016) TU-H-BRC-04: Feasibility of Using Tomo Direct for Pulsed Reduced Dose-Rate Radiotherapy. Medical Physics, 43, 3766. https://doi.org/10.1118/1.4957611

[11] Smilowitz, J.B., Das, I.J., Feygelman, V., Fraass, B.A., Geurts, M., Kry, S.F., et al. (2016) AAPM Medical Physics Practice Guideline 5.a.: Commissioning and QA of Treatment Planning Dose Calculations-Megavoltage Photon and Electron Beams. Journal of Applied Clinical Medical Physics, 17, 457. https://doi.org/10.1120/jacmp.v17i1.6166

[12] Jursinic, P.A. (2013) Dependence of Diode Sensitivity on the Pulse Rate of Delivered Radiation. Medical Physics, 40, 021720. https://doi.org/10.1118/1.4788763

[13] Ahmed, S., Nelms, B., Kozelka, J., Zhang, G., Moros, E. and Feygelman, V. (2016) Validation of an Improved Helical Diode Array and Dose Reconstruction Software Using TG-244 Datasets and Stringent Dose Comparison Criteria. Journal of Applied Clinical Medical Physics, 17, 6414. https://doi.org/10.1120/jacmp.v17i6.6414

[14] Gao, S., Balter, P.A., Rose, M. and Simon, W.E. (2016) A Comparison of Methods for Monitoring Photon Beam Energy Constancy. Journal of Applied Clinical Medical Physics, 17, 242-253. https://doi.org/10.1120/jacmp.v17i6.6454

[15] Sun Nuclear Corporation (2016) IC Profiler Reference Guide. Sun Nuclear Corporation, Melbourne

[16] Schuirmann, D.L. (1981) On Hypothesis Testing to Determine If the Mean of a Normal Distribution Is Contained in a Known Interval. Biometrics, 37, 617.

[17] Westlake, W.J. (1981) Response to T.B.L. Kirkwood: Bioequivalence Testing-A Need to Rethink. Biometrics, 37, 589-594. https://doi.org/10.2307/2530573

[18] Yuen, K.K. and Dixon, W.J. (1973) The Approximate Behavior and Performance of the Two-Sample Trimmed T. Biometrika, 60, 369-374.

https://doi.org/10.1093/biomet/60.2.369

[19] Yuen, K.K. (1974) The Two-Sample Trimmed T for Unequal Population Variances. Biometrika, 61, 165-170. https://doi.org/10.1093/biomet/61.1.165

[20] Beaton, A.E. and Tukey, J.W. (1974) The Fitting of Power Series, Meaning Polynomials, Illustrated on Band-Spectroscopic Data. Technometrics, 16, 147-185. https://doi.org/10.1080/00401706.1974.10489171

[21] Yohai, V.J. (1987) High Breakdown-Point and High Efficiency Estimates for Regression. The Annals of Statistics, 15, 642-665. https://doi.org/10.1214/aos/1176350366 
[22] Koller, M. and Stahel, W.A. (2011) Sharpening Wald-Type Inference in Robust Regression for Small Samples. Computational Statistics \& Data Analysis, 55, 25042515. https://doi.org/10.1016/j.csda.2011.02.014

[23] Low, D.A., Harms, W.B., Mutic, S. and Purdy, J.A. (1998) A Technique for the Quantitative Evaluation of Dose Distributions. Medical Physics, 25, 656-661. https://doi.org/10.1118/1.598248

[24] Shende, R., Gupta, G., Patel, G. and Kumar, S. (2016) Commissioning of TrueBeam TM Medical Linear Accelerator: Quantitative and Qualitative Dosimetric Analysis and Comparison of Flattening Filter (FF) and Flattening Filter Free (FFF) Beam. International Journal of Medical Physics, Clinical Engineering and Radiation Oncology, 5, 51-69. https://doi.org/10.4236/ijmpcero.2016.51006

[25] Klein, E.E., Hanley, J., Bayouth, J., Yin, F.F., Simon, W., Dresser, S., et al. (2009) Task Group 142 Report: Quality Assurance of Medical Accelerators. Medical Physics, 36, 4197-4212. https://doi.org/10.1118/1.3190392

Submit or recommend next manuscript to SCIRP and we will provide best service for you:

Accepting pre-submission inquiries through Email, Facebook, LinkedIn, Twitter, etc. A wide selection of journals (inclusive of 9 subjects, more than 200 journals)

Providing 24-hour high-quality service

User-friendly online submission system

Fair and swift peer-review system

Efficient typesetting and proofreading procedure

Display of the result of downloads and visits, as well as the number of cited articles Maximum dissemination of your research work

Submit your manuscript at: http://papersubmission.scirp.org/

Or contact ijmpcero@scirp.org 